\title{
The Pathological Role of Astrocytic MAOB in Parkinsonism Revealed by Genetic Ablation and Over-expression of MAOB
}

\author{
Heeyoung $\mathrm{An}^{1,2}$, Jun Young $\mathrm{Heo}^{3 *}$, C. Justin Lee ${ }^{1,2 *}$ and Min-Ho Nam ${ }^{4 *}$ \\ ${ }^{1}$ KU-KIST Graduate School of Converging Science and Technology, Korea University, Seoul 02841, \\ ${ }^{2}$ Center for Cognition and Sociality, Institute for Basic Science (IBS), Daejeon 34126, \\ ${ }^{3}$ Department of Medical Science, College of Medicine, Chungnam National University, Daejeon 35015, \\ ${ }^{4}$ Brain Science Institute, Korea Institute of Science and Technology (KIST), Seoul 02792, Korea
}

The cause of Parkinson's disease has been traditionally believed to be the dopaminergic neuronal death in the substantia nigra pars compacta (SNpc). This traditional view has been recently challenged by the proposal that reactive astrocytes serve as key players in the pathology of Parkinson's disease through excessive GABA release. This aberrant astrocytic GABA is synthesized by the enzymatic action of monoamine oxidase B (MAOB), whose pharmacological inhibition and gene-silencing are reported to significantly alleviate parkinsonian motor symptoms in animal models of Parkinson's disease. However, whether genetic ablation and over-expression of MAOB can bidirectionally regulate parkinsonian motor symptoms has not been tested. Here we demonstrate that genetic ablation of MAOB blocks the MPTP-induced augmentation of astrocytic GABA-mediated tonic inhibition of neighboring dopaminergic neurons as well as parkinsonian motor symptoms, indicating the necessity of MAOB for parkinsonian motor symptoms. Furthermore, we demonstrate that GFAP-MAOB transgenic mice, in which MAOB is over-expressed under the GFAP promoter for astrocyte-specific over-expression, display exacerbated MPTP-induced tonic inhibition and parkinsonian motor symptoms compared to wild-type mice, indicating the importance of astrocytic MAOB for parkinsonian motor symptoms. Our study provides genetic pieces of evidence for the causal link between the pathological role of astrocytic MAOB-dependent tonic GABA synthesis and parkinsonian motor symptoms.

Key words: Astrocyte, MAOB, MPTP, Parkinson's disease, Tonic GABA

\section{INTRODUCTION}

Parkinson's disease (PD) is the second-most prevalent neurodegenerative disease with features of irreversible motor dysfunction, including bradykinesia, rigidity, resting tremor, and postural instability. These motor symptoms are believed to be caused by the death of dopaminergic (DA) neurons in substantia nigra pars

Submitted April 9, 2021, Revised April 23, 2021,

Accepted April 23,2021

* To whom correspondence should be addressed.

Jun Young Heo, TEL: 82-42-580-8221, FAX: 82-42-580-8121

e-mail: junyoung3@gmail.com

C. Justin Lee, TEL: 82-42-878-9150, FAX: 82-42-878-9151

e-mail: cjl@ibs.re.kr

Min-Ho Nam, TEL: 82-2-958-6421, FAX: 82-2-958-7034

e-mail:dr.namminho@kist.re.kr compacta (SNpc) [1]. The current pharmacological treatments mainly focus on augmenting dopamine signaling by supplying dopamine precursors (levodopa) and dopamine receptor agonists [2] However, these therapeutic strategies have shown several critical limitations, such as the inability to modify the disease progression and severe adverse events, including levodopa-induced dyskinesia $[2,3]$. These limitations can be attributed to an incomplete understanding of PD pathophysiology.

Recently, reactive astrocytes have received special attention as key players in PD pathophysiology [4-10]. Reactive astrocytes are observed along with DA neuronal death in the SNpc of PD patients $[4,8,11,12]$ as well as in several kinds of animal models of PD, including not only toxin-induced models like MPTP and 6-OHDA models but also genetic models [11-13]. Particularly, our previous report demonstrated that reactive astrocytes express
Copyright (c) Experimental Neurobiology 2021. www.enjournal.org
This is an Open Access article distributed under the terms of the Creative Commons Attribution Non-Commercial License (http://creativecommons.org/licenses/by-nc/4.0) which permits unrestricted non-commercial use, distribution, and reproduction in any medium, provided the original work is properly cited. 
a high level of $\mathrm{MAOB}$, which is responsible for aberrant GABA synthesis in the SNpc of various PD animal models [10]. The astrocytic GABA tonically inhibits neighboring DA neuronal activity, causing nigrostriatal DA deficiency and parkinsonian motor symptoms. In the same report, we further demonstrated that the pharmacological and gene-silencing of MAOB significantly modify the disease progression and alleviates the motor symptoms [10]. However, whether genetic ablation and over-expression of $\mathrm{MAOB}$ can bidirectionally regulate parkinsonism has not been tested. In the current study, we tested the hypothesis that astrocytic MAOB causes aberrant tonic inhibition of SNpc DA neurons and parkinsonian motor symptoms using the MAOB-knockout (MAOB-KO) and astrocytic MAOB overexpression (GFAP-MAOB) mice.

\section{MATERIALS AND METHODS}

\section{Animals}

All mice were kept in a temperature- and humidity-controlled environment with a 12-h light-dark cycle (lights on at 7 a.m.) and had free access to food and water. All animal care and handling were performed according to the directives of the Animal Care and Use Committee of the Institutional Animal Care and Use Committee of KIST (Seoul, Korea; Approval No. 2018-060). 10-week-old male C57BL/6 mice, 10-week-old male MAOB$\mathrm{KO}$ and WT littermate mice (129 strain), and 10-week-old male GFAP-MAOB and WT littermate mice (C57BL/6 strain) were used.

\section{MPTP mouse model}

All MPTP experiments used the acute regimen consisting of four times intraperitoneal injection of MPTP-HCl (M0896, Sigma Aldrich, $2 \mathrm{mg} / \mathrm{ml}$ in saline, $20 \mathrm{mg} / \mathrm{kg}$ for one injection) in a day with $2 \mathrm{~h}$ intervals. For MPTP administration to GFAP-MAOB mice, $75 \%$ dose of MPTP was injected with the same protocol because $100 \%$ dose of MPTP was mostly lethal for GFAP-MAOB mice. MPTP use and safety precautions were as described [14].

\section{Vertical grid test}

The vertical grid test was performed as described in the previous study [15]. Briefly, a mouse was gently placed inside the apparatus at $3 \mathrm{~cm}$ from the top, facing upward, and was allowed to turn around and climb down after 2-day habituation. The whole session of the vertical grid test was recorded. The time for the whole session, the time for turning around, and the ratio of failure steps out of total steps were calculated.

\section{Measurements of MAOB enzyme activity}

$\mathrm{MAOB}$ activity in nigrostriatal tissues of $\mathrm{MAOB} \mathrm{KO}, \mathrm{WT}$, and GFAP-MAOB mice was determined using a commercially available kit (A12214; Thermo Fisher Scientific), following the manufacturer's protocol. Lysates were prepared from each region of the transgenic mouse brain by centrifuging at 16,000 rpm after homogenization with RIPA lysis buffer with phosphatase and protease inhibitor cocktail (Roche, Switzerland). Protein concentration in the supernatant was determined using a Bradford-based assay (Bio-Rad Laboratories, Hercules, CA, USA), and samples were diluted to $500 \mathrm{mg}$ per $2 \mathrm{ml}$ total volume with reaction buffer. Samples were pre-incubated for $30 \mathrm{~min}$ at room temperature with the specific MAOB inhibitor, pargyline hydrochloride $(1 \mu \mathrm{M})$. After incubation, samples were added to individual wells of a 96well microplate. The fluorometric assay was initiated by adding $100 \mu$ of a reaction mixture containing Amplex Red reagent (400 $\mu \mathrm{M}$ ), horseradish peroxidase (HRP; $2 \mathrm{U} / \mathrm{ml}$ ), and benzylamine (2 $\mathrm{mM}$ ), a specific substrate of MAOB. Plates were incubated for 30 $\mathrm{min}$ at room temperature, protected from light, and fluorescence was measured at excitation and emission wavelengths of $550 \mathrm{~nm}$ and $590 \mathrm{~nm}$, respectively, using a microplate fluorometer (Life Sciences, Manchester, UK). $\mathrm{H}_{2} \mathrm{O}_{2}(10 \mu \mathrm{M})$ was used as a positive control, and reaction buffer alone was used as a negative control.

\section{Tonic GABA recording}

$\mathrm{GABA}_{\mathrm{A}}$-receptor-mediated current in $\mathrm{SNpc}$ was measured as previously described [10]. Prior to recording, we prepared 300 $\mu \mathrm{m}$-thick SNpc-containing slices from acutely prepared mouse brains. The brain slices were incubated at room temperature for at least $1 \mathrm{~h}$ in aCSF solution: $126 \mathrm{NaCl}, 24 \mathrm{NaHCO}_{3}, 1 \mathrm{NaH}_{2} \mathrm{PO}_{4}, 0.5$ ascorbic acid, $2.5 \mathrm{KCl}, 2.5 \mathrm{CaCl}_{2}, 2 \mathrm{MgCl}_{2}$, and $10 \mathrm{D}$-(+)-glucose (in $\mathrm{mM}), \mathrm{pH}$ 7.4. For recording, a slice was placed in a recording chamber that was continuously perfused with aCSF solution. The slicing chamber was mounted on the stage of an upright Olympus microscope and visualized with a 60x water immersion objective $(\mathrm{NA}=0.90)$ with infrared differential interference contrast optics and a CCD camera, using Imaging Workbench software (Indec BioSystems, CA, USA). Whole-cell recordings were performed from DA cell somata located in the SNpc. The holding potential was $-60 \mathrm{mV}$. Pipette resistance was typically $6 \sim 8 \mathrm{M} \Omega$ and the pipette was filled with an internal solution: $135 \mathrm{CsCl}, 4 \mathrm{NaCl}, 0.5$ $\mathrm{CaCl}_{2}$, 10 HEPES, 5 EGTA, 2 Mg-ATP, $0.5 \mathrm{Na}_{2}$-GTP, 10 QX-314 (in $\mathrm{mM}$ ), $\mathrm{pH}$ adjusted to 7.2 with $\mathrm{CsOH}$ (278 $285 \mathrm{mOsmol}$ ). Before measuring tonic current, the baseline current was stabilized with D-AP5 $(50 \mu \mathrm{M})$ and CNQX $(20 \mu \mathrm{M})$. Electrical signals were digitized and sampled at 50-ms intervals with Digidata 1320A and Multiclamp 700B amplifier (Molecular Devices) using pCLAMP 

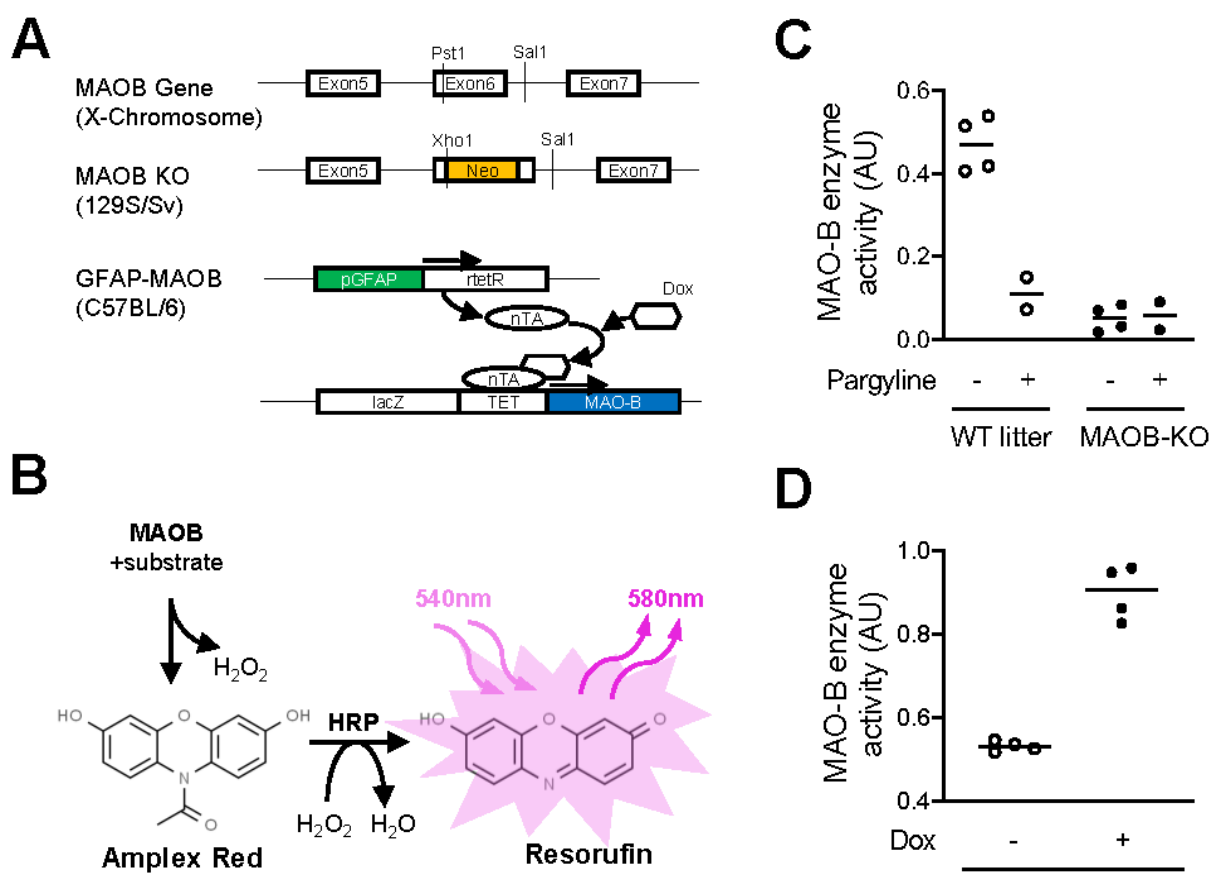

D

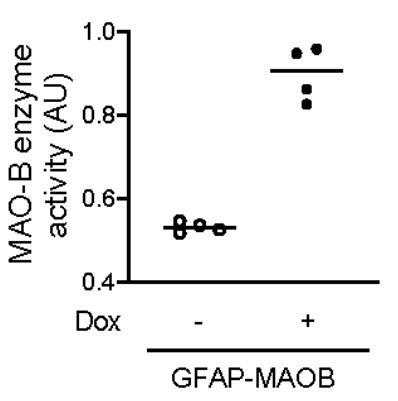

Fig. 1. Validation of MAOB-KO and GFAP-MAOB transgenic mice. (A) Schematic diagram of MAOB-KO and GFAP-MAOB transgenic mice. (B) Schematic diagram of MAOB enzyme activity assay. (C) Quantification of MAOB enzyme activity in MAOB-KO mice and WT littermates. Pargyline is used as a selective MAOB inhibitor ( $\mathrm{N}=4,2,4$, and 2 mice for each group). (D) Quantification of MAOB enzyme activity in doxycycline-treated or nontreated GFAP-MAOB mice ( $\mathrm{N}=4$ mice for each group).

10.2 software. Data were filtered at $2 \mathrm{kHz}$. The amplitude of tonic GABA current was measured by the baseline shift after bicuculline $(50 \mu \mathrm{M})$ administration using the Clampfit program. Frequency and amplitude of spontaneous inhibitory postsynaptic currents (sIPSCs) before bicuculline application was detected and measured by Minianalysis (Synaptosoft, NJ, USA).

\section{Statistical analyses}

Statistical analyses were performed using Prism 9 (GraphPad Software). Differences between two different groups were analyzed with the two-tailed Student's unpaired t-test. For comparison of multiple groups with two independent variables, two-way ANOVA with Tukey's multiple comparison test was assessed. Data from multiple independent experiments was assumed to be normal variance. $\mathrm{p}<0.05$ was considered to indicate statistical significance throughout the study. The significance level is represented as asterisks $\left({ }^{*} \mathrm{p}<0.05,{ }^{* *} \mathrm{p}<0.01,{ }^{* * *} \mathrm{p}<0.001\right.$; ns, not significant). All data were presented as mean \pm SEM. No statistical method was used to predetermine sample size. Sample sizes were determined empirically based on our previous experiences or the review of similar experiments in the literature. Prior to the administration of neurotoxins, animals were randomly and evenly allocated to each experimental group. However, investigators were not blinded to group allocation during experiments or to outcome assessments.

\section{RESULTS AND DISCUSSION}

\section{Validation of MAOB-KO and GFAP-MAOB transgenic mice}

To study the pathological role of MAOB in PD pathology of the MPTP model, we utilized two transgenic mouse lines, MAOB-KO and GFAP-MAOB (Fig. 1A). GFAP-MAOB transgenic mice were reported to over-express $\mathrm{MAOB}$ in astrocytes in a doxycyclinedependent manner [9]. We first validated the enzymatic activity of $\mathrm{MAOB}$ of nigrostriatal tissues in these mouse lines by performing MAOB enzyme assay (Fig. 1B), as previously described [10]. We found that the MAOB enzyme activity of MAOB-KO mice was markedly lower than that of the wild-type (WT) littermates. The $\mathrm{MAOB}$ enzyme activity of MAOB-KO was not further reduced by treatment with pargyline, a selective MAOB inhibitor (Fig. 1C). We also found that the MAOB enzyme activity of doxycycline-treated GFAP-MAOB mice was markedly higher than that of vehicletreated GFAP-MAOB mice (Fig. 1D). These findings indicate that MAOB-KO mice and GFAP-MAOB mice can be utilized for investigating the role of $\mathrm{MAOB}$. 
A

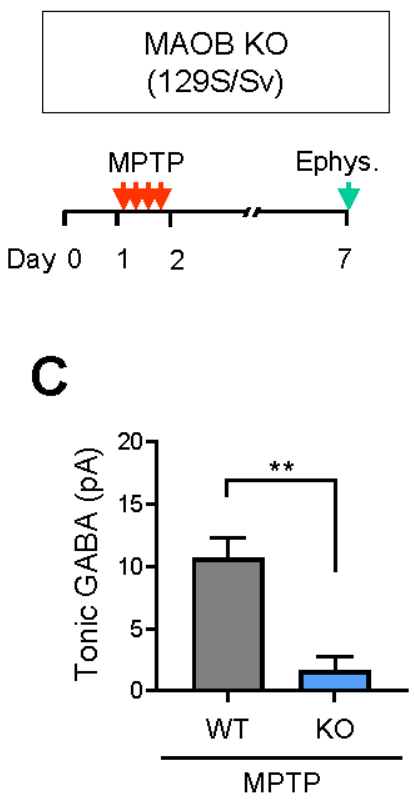

F
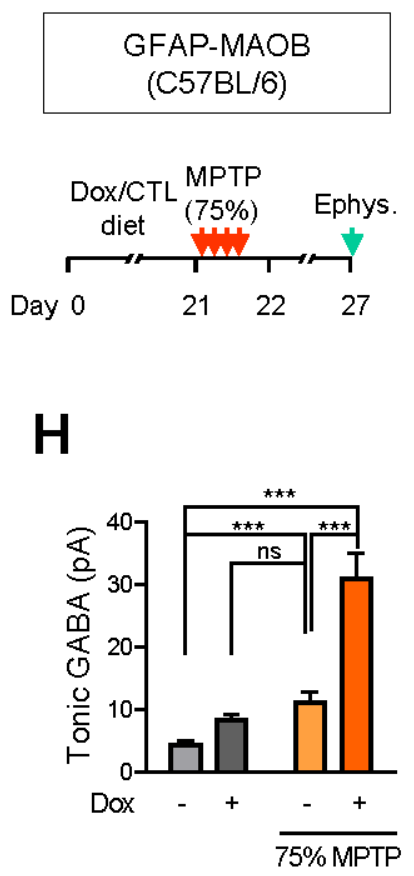

B MPTP

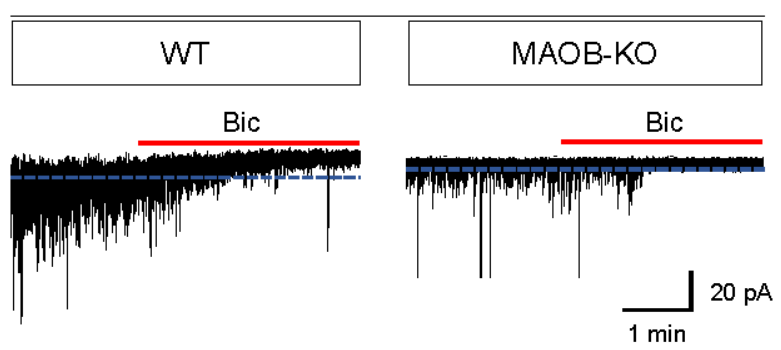

D

E
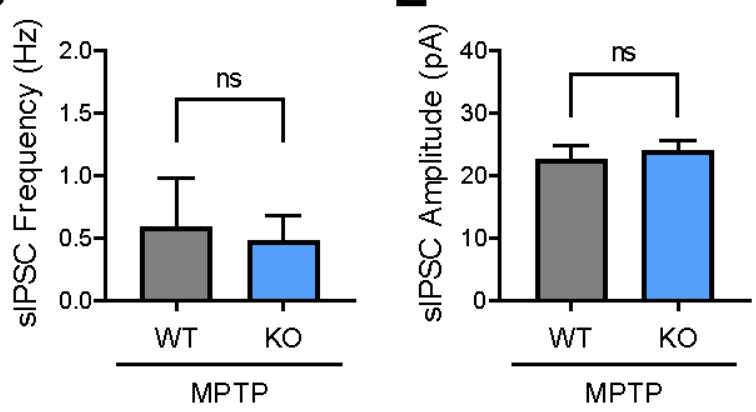

G

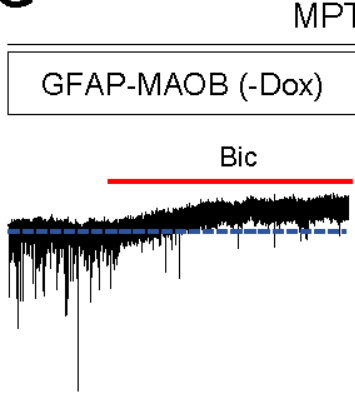

MPTP $(75 \%)$

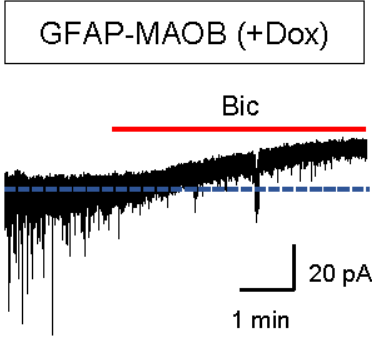

J
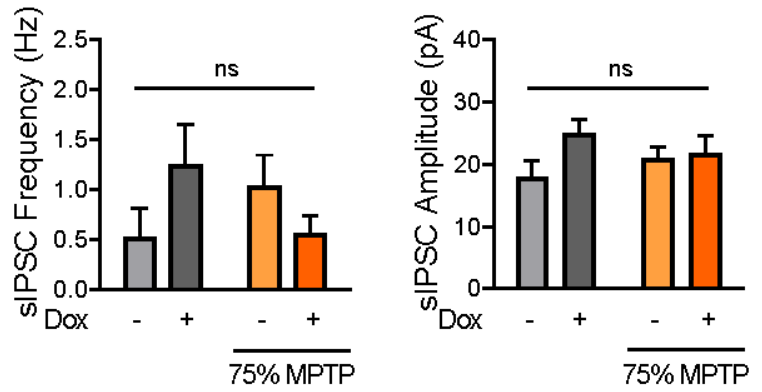

Fig. 2. Astrocytic MAOB is responsible for MPTP-induced aberrant tonic inhibition. (A) Experimental schedule of tonic GABA recording from SNpc DA neurons of MPTP-treated MAOB-KO or WT littermate mice. (B) Representative traces of tonic GABA currents revealed by bath application of bicuculline (Bic, $50 \mathrm{mM}$ ) (C) Quantification of tonic GABA current ( $\mathrm{n}=10$ and 12 cells from 5 mice for each group). (D, E) Quantification of sIPSC frequency and amplitude. (F) Experimental schedule of tonic GABA recording from SNpc DA neurons of GFAP-MAOB mice treated with 75\% dose of MPTP. $(\mathrm{G})$ Representative traces of tonic GABA currents. (H) Quantification of tonic GABA current $(\mathrm{n}=5,4,11$, and 10 cells for each group from 4, 3, 6, and 5 mice, respectively). (I, J) Quantification of sIPSC frequency and amplitude. For all figures, mean \pm SEM; ns, non-significance; ${ }^{* *} \mathrm{p}<0.01,{ }^{* * *} \mathrm{p}<0.001$ assessed by Student's t-test (C E) or two-way ANOVA with Tukey (H J). 
A

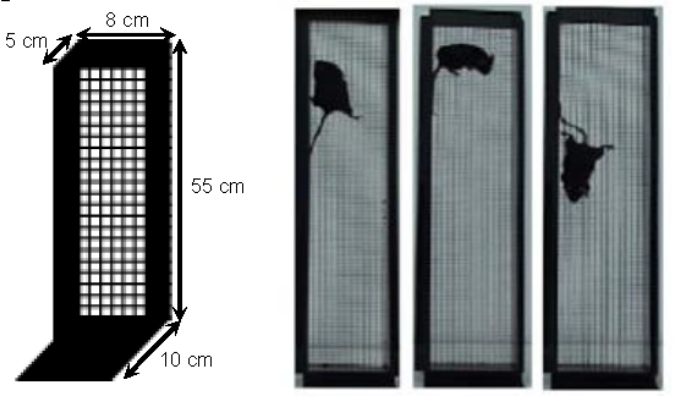

B

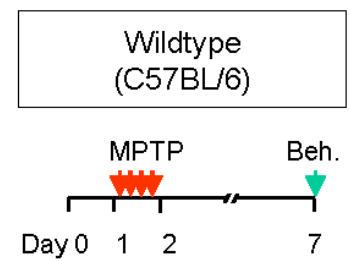

F

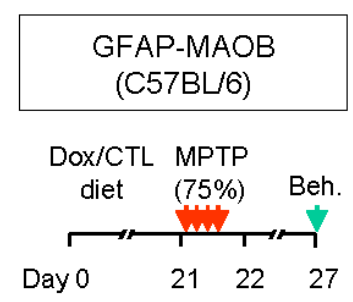

J

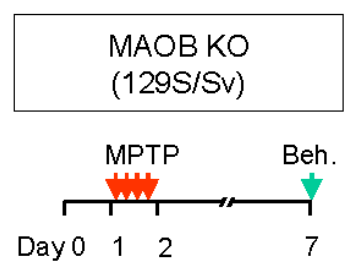

C

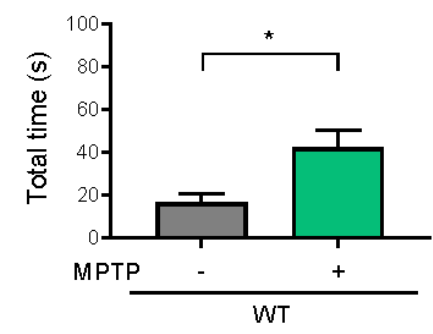

G

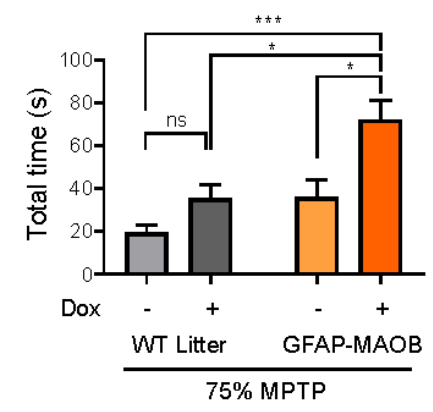

K

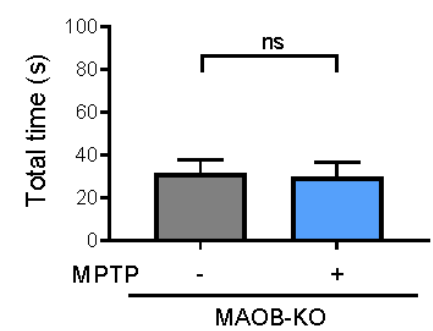

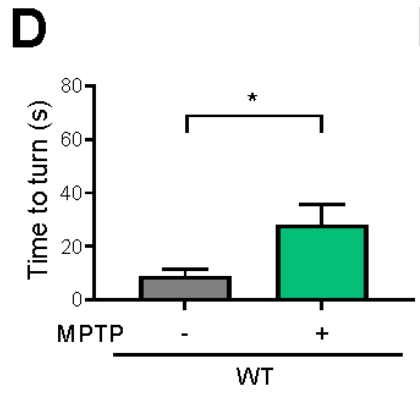

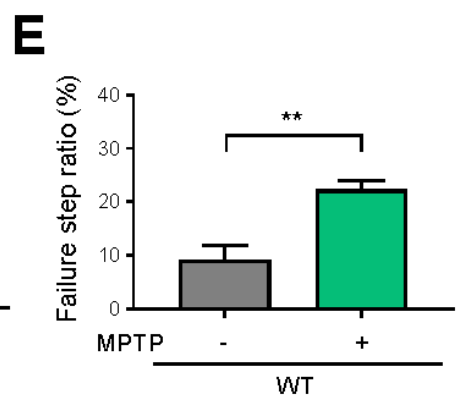

H
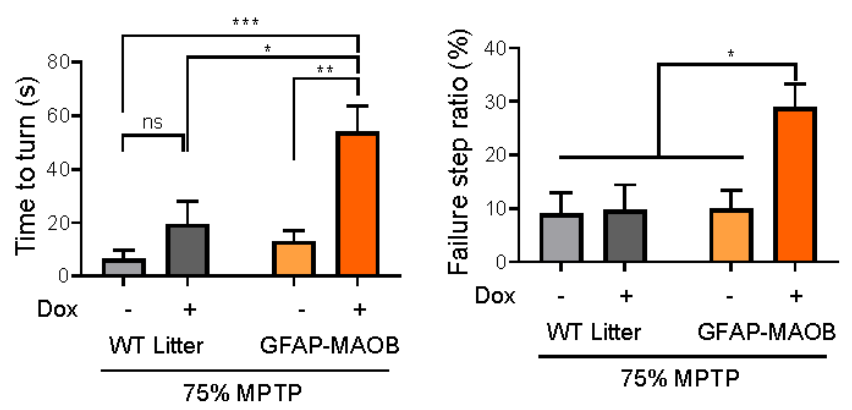

L

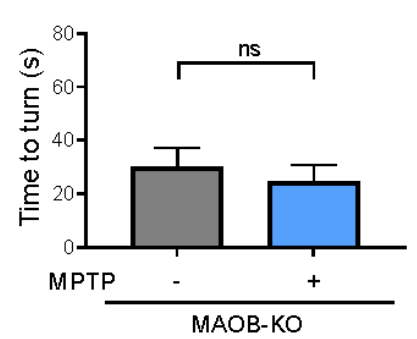

\section{M}

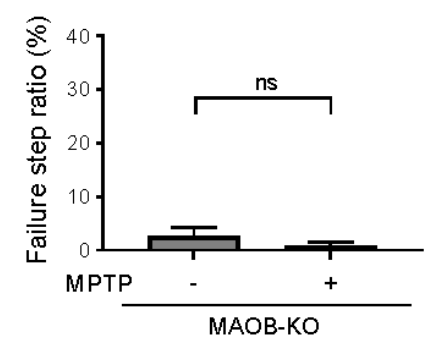

Fig. 3. Astrocytic MAOB is responsible for MPTP-induced parkinsonian motor symptoms. (A) Schematic diagram of vertical grid test. (B) Experimental schedule of vertical grid test after MPTP- or vehicle-treatment in WT mice. (C-E) Quantification of total time, time to turn, and failure step ratio (N=6 mice for each group). (F) Experimental schedule of vertical grid test for GFAP-MAOB transgenic mice treated with 75\% dose of MPTP. (G-I) Quantification of total time, time to turn, and failure step ratio ( $\mathrm{N}=5,4,6$, and 6 mice for each group). (J) Experimental schedule of vertical grid test after MPTPor vehicle-treatment in MAOB-KO mice. (K-M) Quantification of total time, time to turn, and failure step ratio ( $\mathrm{N}=6$ mice for each group). For all figures, mean \pm SEM; ns, non-significance; ${ }^{*} \mathrm{p}<0.05,{ }^{* *} \mathrm{p}<0.01,{ }^{* * *} \mathrm{p}<0.001$ assessed by Student's t-test $(\mathrm{C} \sim \mathrm{E}, \mathrm{K} \sim \mathrm{M})$ or two-way ANOVA with Tukey $(\mathrm{G} \sim \mathrm{I})$. 


\section{Astrocytic MAOB for tonic inhibition of SNpc DA neurons in MPTP model}

We previously reported that $\mathrm{MAOB}$ is responsible for astrocytic GABA synthesis, which accounts for tonic inhibition of neighboring neurons in various brain regions, including the hippocampus, cortex, cerebellum, and SNpc [10, 16-20]. Particularly, we demonstrated that the MAOB-dependent tonic inhibition of DA neurons was aberrantly increased in the SNpc of both PD patients and animal PD models [10]. Based on these previous findings, we tested whether genetic ablation of MAOB blocks MPTP-mediated increase of tonic inhibition by performing whole-cell patch-clamp experiments (Fig. 2A), as previously described [10]. We found that MPTP-treated MAOB-KO mice showed significantly lower tonic GABA current compared to MPTP-treated WT littermates (Fig. $2 \mathrm{~B}, \mathrm{C}$ ). On the other hand, the amplitude and frequency of the spontaneous inhibitory postsynaptic currents (sIPSC) were not different between the two groups (Fig. 2D, E). These findings indicate that MAOB is necessary for augmented tonic GABA inhibition in the MPTP PD model.

We next tested whether astrocyte-specific over-expression of the $\mathrm{MAOB}$ gene further increases the tonic inhibition of SNpc DA neurons in the MPTP PD model (Fig. 2F). We first tested if astrocyte-specific MAOB overexpression increases the tonic GABA current without MPTP treatment. We found that GFAP-MAOB overexpression showed a non-significant but increasing tendency of tonic GABA current. Next, we tested the effect of GFAP-MAOB overexpression in the mice treated with MPTP. MAOB is known to mediate the conversion of MPTP to neurotoxin MPP+, and $\mathrm{H}_{2} \mathrm{O}_{2}$ is produced from this reaction. MAOB overexpression can excessively catalyze the conversion of MPTP, leading to aberrant MPP+ and $\mathrm{H}_{2} \mathrm{O}_{2}$, causing serious damages throughout the brain. Therefore, we treated the animal with only 75\% dose of MPTP rather than $100 \%$ dose, which was mostly lethal to doxycycline-treated GFAP-MAOB transgenic mice. We found that MPTP-treated doxycycline-mediated MAOB over-expression in GFAP-MAOB mice showed a significantly higher tonic GABA current compared to other control groups, including doxycycline-untreated GFAPMAOB mice and WT littermates (Fig. 2G, H). On the other hand, we could not find any significant differences in the amplitude and frequency of sIPSC across the groups (Fig. 2I, J). These findings indicate that $\mathrm{MAOB}$ overexpression exacerbates the augmentation of tonic GABA inhibition in the MPTP PD model.

\section{Astrocytic MAOB for parkinsonian motor symptoms in MPTP model}

We previously demonstrated that the GFAP-MAOB overexpression disrupted motor coordination (Woo et al., PNAS, 2018). To further investigate whether the MAOB-mediated tonic inhibition is responsible for parkinsonian motor symptoms in the MPTP PD model, we performed a vertical grid test (Fig. 3A) which is previously validated for evaluating the motor dysfunction in this animal model [15]. We first confirmed that the MPTP-induced motor dysfunction was well manifested by the increased total time, increased time-to-turn, and increased failure step ratio from the vertical grid test (Fig. 3B-E). We also tested whether GFAP-MAOB overexpression further exacerbates the parkinsonian motor dysfunction in the MPTP PD model (Fig. 3F). We found that MPTP $(75 \%)$ treatment caused more severe parkinsonian motor dysfunction in the doxycycline-treated GFAP-MAOB mice than other control groups (Fig. 3G-I), indicating that MAOB overexpression exacerbates the parkinsonian motor dysfunction in the MPTP PD model. We also tested if genetic ablation of MAOB blocks MPTP-induced parkinsonian motor symptoms using MAOB-KO mice. We found that MAOB-KO mice did not show such MPTPinduced motor dysfunction (Fig. 3J-M), indicating that MAOB is necessary for parkinsonian motor dysfunction in the MPTP PD model. Taken together, these findings strongly suggest that the MAOB-mediated tonic inhibition accounts for parkinsonian motor symptoms in the MPTP PD model.

In summary, we demonstrated the causal relationship between MAOB-dependent tonic inhibition and parkinsonian motor dysfunction in the MPTP PD model. These findings are in line with our previous findings that aberrant tonic inhibition is critical for parkinsonian motor dysfunction in various animal models of PD [10]. Our current study provides missing genetic pieces of evidence for the causal link between the pathological role of astrocytic MAOB-dependent tonic GABA synthesis and parkinsonian motor symptoms.

\section{ACKNOWLEDGEMENTS}

This work was supported by Korea Institute of Science and Technology Grants (2E30951) and Brain Science Program of the National Research Foundation (NRF) of Korea funded by the Ministry of Science and ICT of Korea (2018M3C7A1056897) to M.H.N.; and Institute for Basic Science (IBS), Center for Cognition and Sociality (IBS-R001-D2) to C.J.L.

\section{REFERENCES}

1. Dickson DW (2012) Parkinson's disease and Parkinsonism: neuropathology. Cold Spring Harb Perspect Med 2:a009258.

2. Urso D, Chaudhuri KR, Qamar MA, Jenner P (2020) Improving the delivery of levodopa in Parkinson's disease: a review 
of approved and emerging therapies. CNS Drugs 34:11491163.

3. LeWitt PA (2015) Levodopa therapy for Parkinson's disease: pharmacokinetics and pharmacodynamics. Mov Disord 30:64-72.

4. Teismann P, Tieu K, Cohen O, Choi DK, Wu DC, Marks D, Vila M, Jackson-Lewis V, Przedborski S (2003) Pathogenic role of glial cells in Parkinson's disease. Mov Disord 18:121129.

5. Liberatore GT, Jackson-Lewis V, Vukosavic S, Mandir AS, Vila M, McAuliffe WG, Dawson VL, Dawson TM, Przedborski S (1999) Inducible nitric oxide synthase stimulates dopaminergic neurodegeneration in the MPTP model of Parkinson disease. Nat Med 5:1403-1409.

6. L'Episcopo F, Tirolo C, Testa N, Caniglia S, Morale MC, Cossetti C, D'Adamo P, Zardini E, Andreoni L, Ihekwaba AE, Serra PA, Franciotta D, Martino G, Pluchino S, Marchetti B (2011) Reactive astrocytes and Wnt/ $\beta$-catenin signaling link nigrostriatal injury to repair in 1-methyl-4-phenyl-1,2,3,6tetrahydropyridine model of Parkinson's disease. Neurobiol Dis 41:508-527.

7. Joe EH, Choi DJ, An J, Eun JH, Jou I, Park S (2018) Astrocytes, microglia, and Parkinson's disease. Exp Neurobiol 27:77-87.

8. Hirsch EC, Breidert T, Rousselet E, Hunot S, Hartmann A, Michel PP (2003) The role of glial reaction and inflammation in Parkinson's disease. Ann NY Acad Sci 991:214-228.

9. Mallajosyula JK, Kaur D, Chinta SJ, Rajagopalan S, Rane A, Nicholls DG, Di Monte DA, Macarthur H, Andersen JK (2008) MAO-B elevation in mouse brain astrocytes results in Parkinson's pathology. PLoS One 3:e1616.

10. Heo JY, Nam MH, Yoon HH, Kim J, Hwang YJ, Won W, Woo DH, Lee JA, Park HJ, Jo S, Lee MJ, Kim S, Shim JE, Jang DP, Kim KI, Huh SH, Jeong JY, Kowall NW, Lee J, Im H, Park JH, Jang BK, Park KD, Lee HJ, Shin H, Cho IJ, Hwang EM, Kim Y, Kim HY, Oh SJ, Lee SE, Paek SH, Yoon JH, Jin BK, Kweon GR, Shim I, Hwang O, Ryu H, Jeon SR, Lee CJ (2020) Aberrant tonic inhibition of dopaminergic neuronal activity causes motor symptoms in animal models of Parkinson's disease. Curr Biol 30:276-291.e9.

11. Sun L, Shen R, Agnihotri SK, Chen Y, Huang Z, Büeler H (2018) Lack of PINK1 alters glia innate immune responses and enhances inflammation-induced, nitric oxide-mediated neuron death. Sci Rep 8:383.

12. Fellner L, Irschick R, Schanda K, Reindl M, Klimaschewski L, Poewe W, Wenning GK, Stefanova N (2013) Toll-like receptor 4 is required for $\alpha$-synuclein dependent activation of microglia and astroglia. Glia 61:349-360.

13. Waak J, Weber SS, Waldenmaier A, Görner K, Alunni-Fabbroni M, Schell H, Vogt-Weisenhorn D, Pham TT, Reumers V, Baekelandt V, Wurst W, Kahle PJ (2009) Regulation of astrocyte inflammatory responses by the Parkinson's diseaseassociated gene DJ-1. FASEB J 23:2478-2489.

14. Jackson-Lewis V, Przedborski S (2007) Protocol for the MPTP mouse model of Parkinson's disease. Nat Protoc 2:141-151.

15. Kim ST, Son HJ, Choi JH, Ji IJ, Hwang O (2010) Vertical grid test and modified horizontal grid test are sensitive methods for evaluating motor dysfunctions in the MPTP mouse model of Parkinson's disease. Brain Res 1306:176-183.

16. Jo S, Yarishkin O, Hwang YJ, Chun YE, Park M, Woo DH, Bae JY, Kim T, Lee J, Chun H, Park HJ, Lee DY, Hong J, Kim HY, Oh SJ, Park SJ, Lee H, Yoon BE, Kim Y, Jeong Y, Shim I, Bae YC, Cho J, Kowall NW, Ryu H, Hwang E, Kim D, Lee CJ (2014) GABA from reactive astrocytes impairs memory in mouse models of Alzheimer's disease. Nat Med 20:886-896.

17. Nam MH, Cho J, Kwon DH, Park JY, Woo J, Lee JM, Lee S, Ko HY, Won W, Kim RG, Song H, Oh SJ, Choi JW, Park KD, Park EK, Jung H, Kim HS, Lee MC, Yun M, Lee CJ, Kim HI (2020) Excessive astrocytic GABA causes cortical hypometabolism and impedes functional recovery after subcortical stroke. Cell Rep 32:107861.

18. Lee S, Yoon BE, Berglund K, Oh SJ, Park H, Shin HS, Augustine GJ, Lee CJ (2010) Channel-mediated tonic GABA release from glia. Science 330:790-796.

19. Yoon BE, Woo J, Chun YE, Chun H, Jo S, Bae JY, An H, Min JO, Oh SJ, Han KS, Kim HY, Kim T, Kim YS, Bae YC, Lee CJ (2014) Glial GABA, synthesized by monoamine oxidase B, mediates tonic inhibition. J Physiol 592:4951-4968.

20. Park JH, Ju YH, Choi JW, Song HJ, Jang BK, Woo J, Chun H, Kim HJ, Shin SJ, Yarishkin O, Jo S, Park M, Yeon SK, Kim S, Kim J, Nam MH, Londhe AM, Kim J, Cho SJ, Cho S, Lee C, Hwang SY, Kim SW, Oh SJ, Cho J, Pae AN, Lee CJ, Park KD (2019) Newly developed reversible MAO-B inhibitor circumvents the shortcomings of irreversible inhibitors in Alzheimer's disease. Sci Adv 5:eaav0316. 